STRUCTURAL BIOLOGY

\section{A racemate to the rescue}

Proc. Natl. Acad. Sci. USA

doi:10.1073/pnas.1422387112

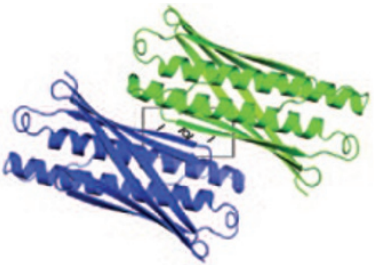

Mycobacterium tuberculosis, the causative agent of tuberculosis, can enter a dormant state within macrophages that is difficult to eliminate and can subsequently cause active infections. Environmental conditions such as hypoxia are thought to be key to initiating dormancy, which is characterized in part by greatly diminished protein synthesis. One of the genes most highly upregulated under these conditions is Rv1738, which encodes a 94-residue protein of unknown function. Bunker et al. envisioned that a crystal structure could be informative about its physiological role. However, crystals could not be obtained with the wild-type sequence or with sequences modified at the termini or using other common 'rescue' strategies. From prior experience, the authors suspected that crystallizing the protein as a racemate would be helpful because additional space groups would be accessible. The authors thus prepared the $\mathrm{L}$ - and $\mathrm{D}$-protein mirror image forms of Rv1738 by total chemical synthesis. The racemic mixture rapidly formed crystals that diffracted to high resolution, and the structure was solved using computational methods that exploited the centrosymmetric crystal form. The structure shows that Rv1738 is a dimer with a six-stranded $\beta$-sheet wrapped around two $\alpha$-helices. Within the crystal, hydrogen bonds between the $\beta$-sheet of the $\mathrm{L}$ - and $\mathrm{D}$-protein forms create an extended ribbon, explaining why the racemic mixture crystallized but the native L-protein did not. With the structure in hand, the authors were able to identify homologs among the S30Ae ribosome-associated proteins, a family that includes cold-shock proteins from Escherichia coli and Vibrio cholerae that inhibit translation during environmental stresses. Modeling further suggested that structural variations between Rv1738 and S30Ae representatives could be accommodated within the ribosomal S30Ae binding site. These data suggest that Rv1738 contributes to the shutdown of ribosomal protein synthesis during the onset of dormancy, and set the stage for more directed study of this activity.
NEUROSCIENCE

\section{An epileptic target}

There are several drugs that are effective for treatment of epilepsy, but in some cases where these fail to prevent seizures, a ketogenic diet has provided effective management of the disease. This diet switches the brain's energy source from glucose to ketone bodies, which suppress seizures via modulation of neuronal ATP-sensitive $\mathrm{K}^{+}\left(\mathrm{K}_{\text {ATP }}\right)$ channels and adenosine $A_{1}$ receptors, but the metabolic events that lead to this suppression are not known. To further explore the connection between metabolism and epilepsy, Sada et al. examined neuronal activity following a switch from glucose to ketone-body utilization. They found that STN cells of the basal ganglia, known to be important for some forms of seizures, are hyperpolarized during the switch in energy source. This hyperpolarization could be completely reversed with lactate, indicating that the hyperpolarization during the switch is due to depletion of glucose and a subsequent lack of conversion into lactate, a neuronal glucose metabolite that is known to be important for regulating various brain functions. Indeed, lactate concentrations in the hippocampus were lower in mice fed a ketogenic diet. Spurred by the idea that the lactate pathway is involved in the hyperpolarization seen during the change in energy source, the authors tested the effects of oxamate, an inhibitor of lactate dehydrogenase (LDH), and found that oxamate treatment also induced hyperpolarization. This could be reversed by addition of the downstream LDH metabolites pyruvate and oxaloacetate as well as by $\mathrm{K}_{\text {ATP }}$ inhibition. The oxamate-induced hyperpolarization occurred only in neuronal cell types in which there is a putative link between the downstream metabolites of LDH and $\mathrm{K}_{\text {ATP }}$ channels. LDH inhibition by oxamate or by knockdown could suppress seizures and epileptiform activity in two mouse models. Finally, the authors found that the anti-epileptic drug stiripentol and a related compound, isosafrole, inhibited LDH activity. These results suggest that LDH inhibition suppresses epilepsy by mimicking the effects of a ketogenic diet and identified the first anti-epileptic drug known to act on a metabolic pathway.
METABOLIC REGULATION

\section{Go fish}

Cell Rep. 10, 694-701 (2015)

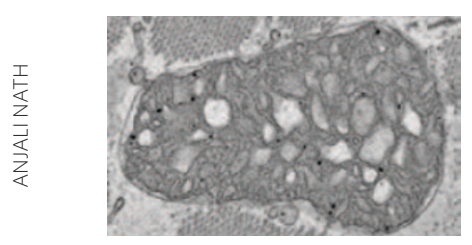

Model organisms such as zebrafish offer unique advantages for chemical screening in terms of cost and ease of screening. Nath et al. wanted to validate the use of zebrafish larvae as a model system for smallmolecule screening to identify regulators of glucose metabolism. As expected, treatment of larvae with anti-diabetic drugs such as insulin and pioglitazone decreased glucose levels. In addition, ablation of liver cells using a tissuespecific nitroreductase transgenic system decreased glucose levels, while similar ablation of pancreatic $\beta$ cells increased glucose levels. With this validation in place, the authors then treated larvae with a library of 13,120 small-molecule compounds to identify those that lowered systemic glucose levels. One of the most effective candidates was alexidine, a known mitochondrial phosphatase PTPMT1 inhibitor. Treatment of larvae with additional, structurally distinct PTPMT1 inhibitors and development of a TALEN-generated ptpmt1 knockout also yielded reduced glucose levels. Alexidine treatment was ineffective in $p t p m t 1$ mutants, confirming that PTPMT1 is the compound's target. To determine the potential substrates of PTPMT1 activity in the mitochondria, Nath et al. analyzed phosphotyrosine- and phosphoserinemodified proteins that were differentially expressed between wild-type and ptpmt1heterozygous larvae. They found that the succinate dehydrogenase (SDH) subunit SDHA, which is involved in TCA and electron transport chain metabolism, was hyperphosphorylated in ptpmt1 mutants. Considering that FGR kinase phosphorylates SDHA, the authors thought that PTPMT1 might reduce SDHA phosphorylation. As expected, PTPMT1 decreased FGR-mediated phosphorylation of SDHA, resulting in diminished SDH activity. Finally, given that alexidine was unable to lower glucose in larvae lacking insulin-producing beta cells, it is possible that PTPMT1 regulation of SDH activity may lower glucose levels by stimulating increased insulin secretion. 